\section{Long term imatinib treatment in pulmonary arterial hypertension}

Pulmonary arterial hypertension (PAH) is a life threatening condition characterised by progressive obliteration of the small pulmonary arteries leading to increased pulmonary arterial resistance and right heart failure. Treatment for PAH has developed in the last few years since the description of new pathways related to the disease. ${ }^{1}$ Recently, short term (6 months) use of imatinib, a platelet derived growth factor (PDGF) receptor antagonist, in combination with maximal $\mathrm{PAH}$ treatment (prostacyclin derivative, endothelin receptor antagonist, and type 5 phosphodiesterase inhibitor) has been shown to improve the haemodynamics and functional capacity in a single case of severe PAH. We here report the first two cases of the long term ( 3 years or more) use of imatinib, as monotherapy or in combination with bosentan, a dual endothelin receptor antagonist.

Case 1 was a 34 year old man with $\mathrm{PAH}$ associated with type I glycogen storage disease. Until 1999 the patient had remained stable with functional class II PAH (New York Heart Association classification) without any specific treatment. In 2002 he presented with an increased white blood cell count at his routine evaluation resulting in the diagnosis of chronic myeloid leukemia, a known late complication of type I glycogen storage disease. The patient remained in functional class II and the haemodynamic pattern showed a trend to worsening without a significant change at the 6 minute walk test (fig 1A). Imatinib was started as first line treatment for leukemia without any associated PAH treatment. During 3 years of imatinib use his leukemia was adequately controlled, functional capacity was sustained, and the haemodynamic profile was improved.

Case 2 was a 65 year old woman with a known diagnosis of chronic myeloid leukemia since 1994 which was satisfactorily controlled with hydroxycarbamide, cytarabine, and interferon. In 1996 she reported dyspnoea on exercise with insidious progression during the next 4 years. In 2000 an echocardiogram showed a right ventricular systolic pressure of $65 \mathrm{~mm} \mathrm{Hg}$. In 2002 she presented with functional class III PAH and was referred. The investigation showed no other condition associated with PAH but significant haemodynamic impairment (fig 1B). Treatment with bosentan was initiated with a good haemodynamic and functional response after 3 months. At that time interferon was withdrawn and imatinib was started as treatment for leukemia. Since then, after more than 3 years of treatment with imatinib and bosentan, there has been functional and haemodynamic improvement (fig 1B).

In these two cases of long term use of imatinib (alone or in combination with bosentan), functional and clinical stabilisation or improvement in PAH were observed. Of note is the progressive increase in cardiac index during the 3 years of treatment with imatinib. Such a progressive increase in cardiac index is certainly an indicator of a good prognosis, as previously discussed in $\mathrm{PAH}^{3}$ PDGF is a potent mitogen that has been related to the chemotaxis and proliferation of pulmonary vascular smooth muscle cells. Its inhibition has been shown to prevent and reverse pulmonary hypertension in experimental models, raising the potential

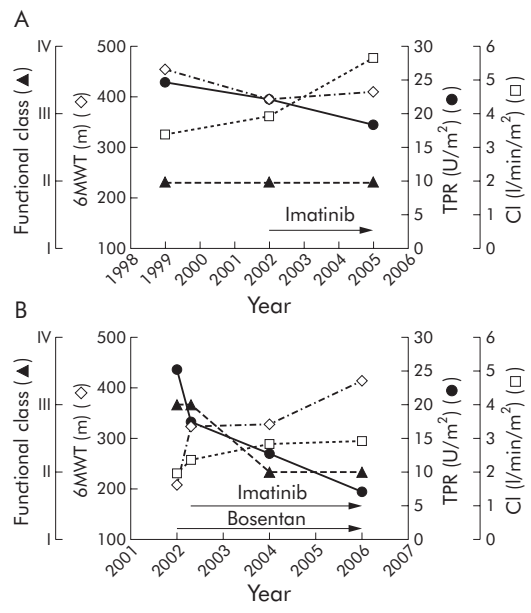

Figure 1 Long term haemodynamic and functional effects of treatment in $(A)$ case 1 and (B) case 2. 6MWT, six minute walk test; TPRi, indexed total pulmonary resistance; $\mathrm{Cl}$, cardiac index; functional class, NYHA classification.

of its use in clinical practice. ${ }^{4}$ Imatinib is approved for the treatment of chronic myeloid leukemia, which was the reason for using it in the two reported cases leading to haematological remission. In parallel, the haemodynamic response to imatinib alone or in combination with bosentan was significant. Although many side effects have already been described with the long term use of imatinib, ${ }^{5}$ only mild anaemia was observed in our first case. We conclude that PDGF inhibition should be tested in future PAH clinical trials in order to establish its safety and efficacy.

R Souza, O Sitbon, F Parent, G Simonneau, M Humbert Centre des Maladies Vasculaires Pulmonaires, UPRES EA2705, INSERM U764, IFR13, Service de Pneumologie et Réanimation respiratoire, Hôpital Antoine-Béclère, Université Paris-Sud, Assistance

Publique - Hôpitaux de Paris, Clamart, France

Correspondence to: Professor M Humbert, Centre des Maladies Vasculaires Pulmonaires, UPRES EA2705 INSERM U764, IFR 13, Service de Pneumologie ef Réanimation Respiratoire, Hôpital Antoine Béclère,

Assistance Publique - Hôpitaux de Paris, Université

Paris-Sud 11, 157 rue de la Porte de Trivaux, 92140 Clamart, France; marc.humbert@abc.aphp.fr

doi: 10.1136/thx.2006.064097

Funding: Chancellerie des Universités de Paris (Legs Poix), Université Paris-Sud 11 and European Respiratory Society, Fellowship number 192.

\section{References}

1 Humbert M, Sitbon $O$, Simonneau G. Treatment of pulmonary arterial hypertension. N Engl J Med 2004;351:1425-36.

2 Ghofrani HA, Seeger W, Grimminger F. Imatinib for the treatment of pulmonary arterial hypertension. N Engl J Med 2005;353:1412-3.

3 Provencher S, Sitbon $O$, Humbert $M$, et al. Longterm outcome with first-line bosentan therapy in idiopathic pulmonary arterial hypertension. Eur Heart J 2006;27:589-95.

4 Schermuly RT, Dony E, Ghofrani HA, et al. Reversal of experimental pulmonary hypertension by PDGF inhibition. J Clin Invest 2005;115:2811-21.

5 Robert C, Soria JC, Spatz A, et al. Cutaneous side-effects of kinase inhibitors and blocking antibodies. Lancet Oncol 2005;6:491-500.

\section{Reduced exercise capacity in a mouse model of asthma}

One of the important clinical features of asthma is the exercise intolerance due to an exacerbation. ${ }^{1}$ Yet, to our knowledge, this end point has never been assessed in animal models of asthma. In a mouse model of chemical induced asthma we found early and late alterations in ventilatory function using unrestrained whole body plethysmography. ${ }^{2-4}$ Partly because this technique has been heavily criticised, ${ }^{5}$ we sought to provide another measure of functional impairment in asthma-namely, exercise endurance. This was done by a simple exercise test in which mice were forced to swim against a limited downwards current; ${ }^{6}$ if they stopped swimming the current pulled them under the water. Swimming endurance time is measured as a proxy of exercise tolerance. Task failure is defined as a period of 5 seconds under water. ${ }^{6}$

The protocol for sensitising and challenging mice was similar to that used previously, $^{2-4}$ with small modifications. Male $\mathrm{BALB} / \mathrm{c}$ mice $( \pm 20 \mathrm{~g}, 6$ weeks old $)$ received dermal applications of $20 \mu \mathrm{l}$ vehicle $(2: 3$ acetone:olive oil) or $0.3 \%$ toluene-2,4-diisocyanate (TDI) on each ear on days 1 and 8 . On day 15 they received an intranasal instillation of $10 \mu \mathrm{l}$ vehicle or $0.1 \%$ TDI in each nostril. Treatment with TDI is indicated as 1 , while treatment with vehicle is indicated as 0 . Thus, the $1 / 1 / 1$ group consists of mice that received dermal applications of TDI (days 1 and 8 ) and an intranasal instillation of TDI (day 15), while the $0 / 0 / 0$ control group consists of mice that received the vehicle on all occasions. All mice had a 5 minute swimming training session on day 13. All swim tests were done in water at $34^{\circ} \mathrm{C}$.

On day 15 resting ventilatory function (enhanced pause (Penh)) of each mouse was recorded by whole body plethysmography (EMKA Technologies, Paris, France) for 5 minutes before and 40 minutes after the intranasal instillation. One hour after instillation the mice were made to swim in groups of 2-3 until exhaustion, as defined above. On day 16 (that is, 22 hours after intranasal instillation) the mice had a second swim test. Two hours later methacholine reactivity was assessed by whole body plethysmography. The measurement of the swimming time was not conducted in a blinded manner, but we are confident that this did not influence the results. All experimental procedures were approved by the local Ethical Committee for Animal Experiments.

Figure 1 shows the early increase in Penh (panel A) and late increase in methacholine reactivity (panel $\mathrm{B}$ ) in mice sensitised and challenged with TDI (group 1/1/1) compared with non-sensitised mice that received TDI $(0 / 0 / 1)$ and control mice $(0 / 0 / 0)$. These changes are similar to those of our previous experiments. ${ }^{2}$ The outcomes of the swimming test parallel those of ventilatory function: in the $1 / 1 / 1$ group endurance was reduced by 13 minutes (panel C) and by 5 minutes (panel D) at the early and late time points, respectively, compared with the 0/0/1 group whose swimming times did not differ from those of the 0/0/0 group. Additionally, the swim time of the control mice was comparable to those of Matsumoto et al. ${ }^{6}$ When looking at responses in individual mice, the magnitude of the impairment in the first swim test correlated (non-parametric Spearman correlations) well with the magnitude of the early increase in Penh $(r=-0.84$, 